\title{
A computationally efficient simulation method for optimizing front contacts of concentrator multijunction solar cells
}

\author{
Chris H. van de Stadt ${ }^{1,2}$, Pilar E. Gonzalez ${ }^{2}$, Harry A. Atwater ${ }^{2}$ and Rebecca Saive ${ }^{1,2}$ \\ ${ }^{1}$ University of Twente, Enschede, 7522, the Netherlands \\ ${ }^{2}$ California Institute of Technology, Pasadena, CA 91125, USA
}

\begin{abstract}
In this work, a novel multidiode model is proposed for optimizing the front grid of multijunction solar cells operating under concentration conditions. The model allows for quickly exploring the maximum achievable efficiency under a wide range of operating conditions and design parameters such as the redirecting capability, period and width of the fingers, the light concentration, and the metal and emitter sheet resistivity. The proposed multidiode model shows to be consistent with experimental data and with more complex modeling approaches such as the simulation program with integrated circuit emphasis (SPICE) model.
\end{abstract}

Index Terms - solar cell front contact grids, device modeling, multijunction solar cells, concentrator photovoltaics.

\section{INTRODUCTION}

By far the majority of power losses in III-V solar cells is occurring at the front contact of the cell [1]. The reason for this is simple: the front side of a solar cell faces a double task. On the one hand it has to transmit as much light as possible in order to maximize the short circuit current. On the other hand, it has to conduct away as many electrons as possible in order to minimize resistive losses. Due to the opto-electronic properties of solids, good electron conductors are generally bad light transmitters and vice versa. This leaves us with a delicate trade-off between transmission and conduction in designing the sun-facing side of a solar cell.

In multijunction solar cells, this trade-off is normally settled by applying a metal grid to the front side of the cell. The various design parameters such as the spacing between the grid lines and the width of the lines are then optimized for a specific incoming light spectrum by means of ray-tracing algorithms and device simulations such as a simulation program with integrated circuit emphasis (SPICE) model [2].

In concentrator applications, in which the incoming light is concentrated onto a small area cell in order to increase efficiency and decrease material costs, this optimization is done over a range of concentrations in order to find the operating point at which the efficiency is maximum.

In order to further improve concentrator multijunction cells efficiencies, efforts are being made to design grid lines that do not reflect all the incoming light away from the cell but instead redirect part of the incoming light towards the active cell area.
The extreme version of such contact are effectively transparent contacts (ETCs) which direct redirect almost all light due to their triangular cross-section and specular silver mirror side wall [3][6]. This further increases the cell's efficiency by allowing more photons to be absorbed and thus increasing the short-circuit current for a given grid coverage. However, as the fraction of the light being redirected to the cell for a certain geometry is often not precisely known, this additional degree of freedom in the design creates an extra variable to be taken into account in modeling such cells.

Running optical and semiconductor models over such a vast parameter space becomes computationally expensive rather fast, creating the need for a computationally cheap and efficient model for optimizing partially redirecting front grids for multijunction solar cells under concentration.

There is a wide variety of approaches for modeling multijunction solar cells under concentration, depending on the modeler's main goal. A single-diode model is the simplest of them and is in some cases sufficient for a rough estimate of the cell's behavior. However, it fails to take into account the effects of distributed resistances, the individual subcell's characteristics and current mismatching between the subcells. Therefore, a single-diode model is unable to cope with changes on the subcell level and is unreliable when a wide range of operating parameters is used.

A multi-diode model takes away some of these limitations by individually taking into account the subcells and connecting them in series. It has been shown that an extension from a single to a multi-diode model improves the modeling accuracy at limited computational costs (which increases linear with the number of subcells). However, just as its single-diode counterpart, multi-diode models don't take into account effects of distributed resistances.

Distributed models such as SPICE are normally used when these distributed effects need to be taken into account. In a distributed SPICE model, the cell is split up in a large number of small spatial elements [7]. Each element has its own set of solar cell parameters such as dark current, series resistance or ideality factor, thus allowing the model to more accurately account for 
variations in local current and voltage due to these spatially varying parameters. Although more accurate, using a SPICE model also enormously increases computational costs as the number of elements used is typically very large and each element is solved individually.

Taking into account the large number of variables - such as grid spacing, amount of redirection, number of grid fingers and concentration factor - over which multijunction concentrator cells need to be modeled in order to optimize the front grid layout, it should be clear that the computational cost of SPICE models is undesirable. In this paper, a more efficient way of modeling multijunction solar cells with partially redirecting front grids will be presented and validated by experimental and SPICE model results. This model is based on a multi-diode model and can account for varying metal resistivity, finger spacing, redirecting capability and finger width. To determine the generated short circuit current density, optical simulations are performed and will be presented in the next section.

\section{OPTICAL MODEL}

For the computational simulation of light interacting with the multijunction cell, multi-scale simulations based on thin film optics combined with ray tracing are performed by PVlighthouse's SunSolve cloud software package [8], [9]. In this software, the cell is built up of a number of layers and films, each defined by a thickness and a material with a certain complex refractive index $(n+i k)$. This results in wavelength and angle dependent coefficients of reflection, transmission and absorption. After these coefficients are calculated, a Monte Carlo ray-tracing simulation is performed by taking a random set of possible rays from a defined incoming spectrum.

Different types of contacts can be designed in SunSolve. Here, we compare rectangular contacts and triangular cross-section effectively transparent contacts (ETCs). A Schematic of these two contact types is shown in Fig. 1a. The contacts run along the $\mathrm{x}$-direction.

Besides the actual cell itself, a typical optical concentrator setup consists of a lens to focus the light onto the cell and an encapsulation to protect the cell from degradation by its outside environment. To simulate this scenario, the set-up shown in Fig. $1 \mathrm{~b}$ is used. It consists of a lens that focusses the light on the cell thereby creating an illumination profile that depends on the position on the cell. In particular, the angle of incidence is less steep the further towards the edges of the solar cell. In the center of the cell the light is incident perpendicular to the cell.

The spatial distribution of the current generation is shown in Fig. 1c with reflecting contacts and Fig. 1d with ETCs. For the current generation it is assumed that each photon absorbed in the active layer generates one electron-hole pair than is collected by the contacts - i.e. the internal quantum efficiency is assumed to be $100 \%$ which is a valid assumption for high quality solar cells. Overall, replacing flat, reflecting fingers by ETCs leads to a 5.8\% enhancement of the peak performance short circuit current. The absorption within the single layers of the 4 junction solar cell is affected in different ways depending on the angle of incidence. The presented results assume that current matching is necessary and therefore, consider the lowest generated current for the overall result. a)

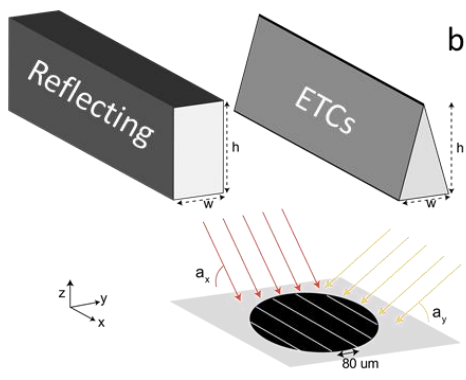

b)

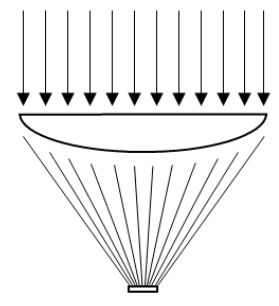

c)

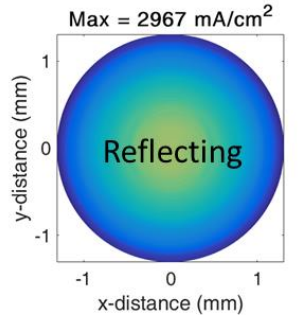

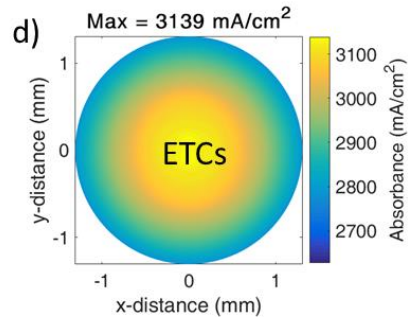

Fig. 1. a) Schematic of the flat, reflecting contacts and of ideal redirecting effectively transparent contacts (ETCs). b) The incident light profile on the cell. All shapes are rotational symmetric. Absorbance in active layers with c) reflecting contacts and d) ETCs.

\section{MULTIDIODE MODEL}

The proposed model uses the electrical circuit of a multijunction (in this case four-junction) cell. This electrical equivalent circuit explicitly takes into account the separate subcells by modelling them individually and connecting them in series by means of a tunnel junction. Each subcell $i$ itself consists of a series resistance $R_{S}$, accounting for resistive losses in the subcell, a shunt resistance $R_{S h}$, accounting for shunt pathways in the subcell which in the case of high efficiency III-V solar cells can be taken to be of infinite value, a current source $I_{L}$ accounting for the light generated current in the subcell, and a diode, accounting for the behavior of a solar cell in the dark. The current of each diode is given by

$$
I_{d}=I_{0}\left(e^{\frac{q V_{d}}{m k_{B} T}}-1\right),
$$

where $I_{d}$ is the current through the diode, $I_{0}$ is the dark saturation current of the diode, $q$ is the electron charge, $m$ the 
diode's ideality factor, $k_{B}$ the Boltzmann constant, $T$ the temperature and $V_{d}$ the voltage across the diode.

Using (1), the current-voltage relationship for each individual subcell can be deduced from electrical circuit theory. Neglecting the -1 term in (1) and taking the shunt resistance to be infinite, it can be shown that the current-voltage relationship for each individual subcell $i$ is

$$
I_{i}=I_{L, i}-I_{0, i} e^{\frac{q\left(V_{i}-I_{i} R_{S, i}\right)}{m_{i} k_{B} T}} .
$$

For the complete cell, all individual resistances will be summed to obtain a total series resistance $R_{\text {tot }}$. The final currentvoltage relationship is then given by equating the currents in all subcells (as they are connected in series) and sum the subcell voltages for each current. A comparison of an experimental and in this way calculated current-voltage relationship for 4 junction concentrator cell from Fraunhofer ISE [10] is shown in Fig. 2.

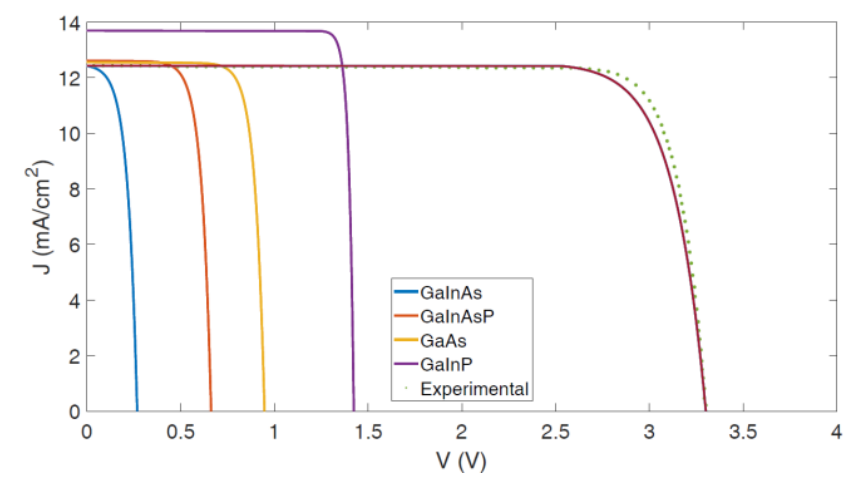

Fig. 2. A comparison of the model's IV curves and an experimentally obtained IV curve.

From this IV-curve, a power conversion efficiency can be calculated. It should be noted that in order to obtain the IV-curve and thus the resulting efficiency in this way, several parameters have to be known. Some of them (such as $I_{0}$ and $m$ ) have to be determined experimentally. Others, however, most notably the lumped series resistance $R_{S}$ and the light generated current $I_{L}$ can be calculated theoretically or taken as an input variable. This allows for exploring a wide variety of front grid designs for multijunction cells under concentration.

For instance, a common design criterion is the maximum achievable efficiency of a concentrator solar cell. Among other things, this is dependent on the used concentration factor, the redirecting capabilities of the finger and how closely these fingers are placed apart from each other. The current model allows us to explore all three of the variables above with short computation time. In Fig. 3 the fill factor and efficiency for a 4 junction concentrator solar cell from Fraunhofer ISE [10] with poor redirection (original cells) and with ETCs is shown. The yellow dots in the fill factor curve represent the experimental data in [10]. It can be seen that for concentration higher than 100 suns the simulation nicely matches the experimental results for the fill factor. With ETCs, high fill factor can be maintained even at high concentration due to the increased contact cross-section and therefore, reduced resistivity. Due to the computational efficiency, these results can be obtained within only a couple of minutes.
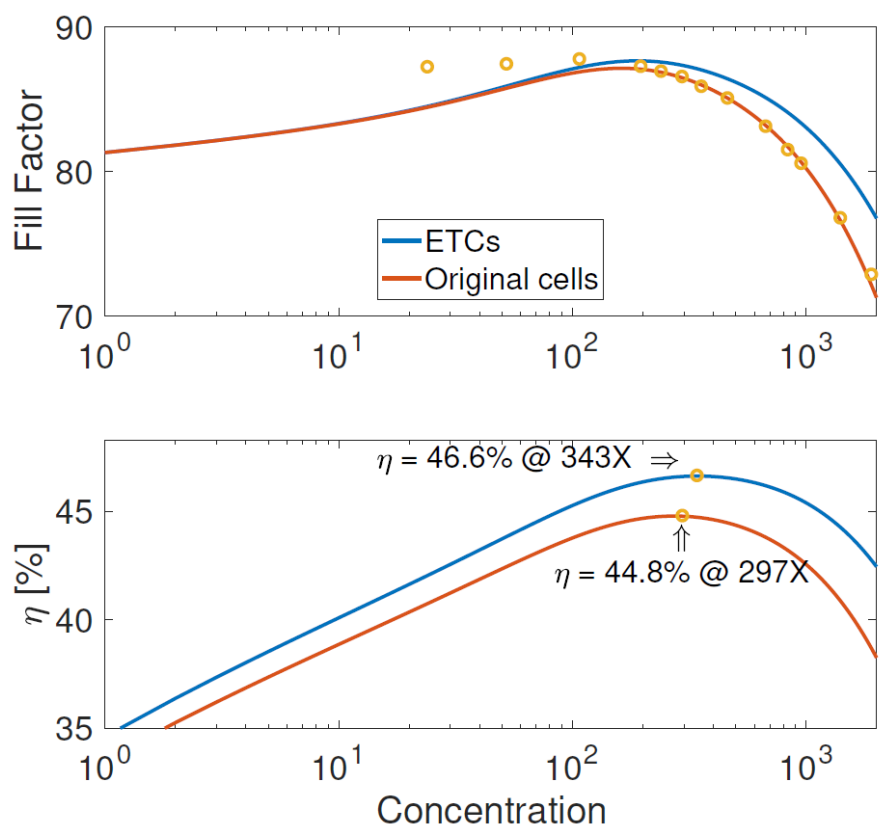

Fig. 3. Concentration dependent efficiency and fill factor simulated for a 4 junction concentrator solar cell from Fraunhofer ISE [10] with poor redirection (original cells) and with ETCs. The yellow dots in the fill factor curve represent the experimental data in [10].

We also performed SPICE model simulations for the same device. The fill factor vs. concentration curves for the multidiode model, the SPICE model, and the experimental result are shown in Fig. 3. It can be seen that the multi-diode model follows the experimental points more closely than the SPICE model. The most important reason for this is that the actual operating conditions and experimental data of the modeled cell can be implemented more directly into the multi-diode model. For instance, for the multi-diode model, the total series resistance is taken directly from the reported IV- and FF curves, whereas the series resistance in the SPICE model is calculated in a bottom-up approach. The bottom up approach is helpful if the experimental cell parameters (such as tunnel diode resistance) are not known. In the multidiode model one would have to guess those parameters based on relevant literature data. If the experimental 
behavior of the cell is already know in detail for one specific front contact configuration, then the multidiode model is superior as those parameters can directly be used.

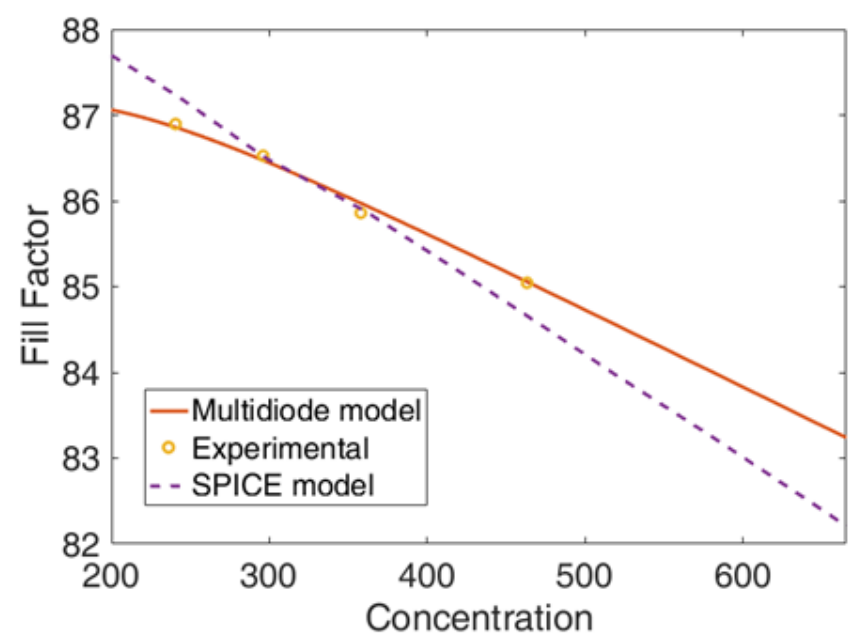

Fig. 3. Comparison of FF obtained from SPICE model, multidiode model, and experimental results taken from[10].

\section{CONCLUSION}

A novel modeling approach has been proposed that allows for a computationally efficient exploration of the design parameters for front grids on multijunction solar cells under concentration. This model is based on a single diode model for each individual subcell of the multijunction stack, combined with a lumped series resistance for the overall cell. The model agrees with experimental data. Using this model, changes in front grid design such as the redirecting capabilities and periodicity of the fingers can be evaluated over a wide range of concentrations with minimum computational effort.

\section{REFERENCES}

[1] M. Steiner, S. P. Philipps, M. Hermle, A. W. Bett, and F. Dimroth, "Validated front contact grid simulation for GaAs solar cells under concentrated sunlight," Progress in Photovoltaics: Research and Applications, vol. 19, no. 1, pp. 73-83, 2011.

[2] M. Steiner, W. Guter, G. Peharz, S. P. Philipps, F. Dimroth, and A. W. Bett, "A validated SPICE network simulation study on improving tunnel diodes by introducing lateral conduction layers," Progress in Photovoltaics: Research and Applications, vol. 20, no. 3, pp. 274-283, 2012.

[3] R. Saive and H. A. Atwater, "Mesoscale trumps nanoscale: metallic mesoscale contact morphology for improved light trapping, optical absorption and grid conductance in silicon solar cells," Optics Express, vol. 26, no. 6, pp. A275-A282, 2018.
[4] R. Saive et al., "Silicon heterojunction solar cells with effectively transparent front contacts," Sustainable Energy \& Fuels, vol. 1, pp. 593-598, 2017.

[5] R. Saive et al., "Effectively Transparent Front Contacts for Optoelectronic Devices," Advanced Optical Materials, vol. 4, no. 10, pp. 1470-1474, 2016.

[6] R. Saive et al., "Effectively transparent contacts (ETCs) for solar cells," in Photovoltaic Specialists Conference (PVSC), 2016 IEEE 43rd, 2016, pp. 3612-3615: IEEE.

[7] L. Nagel and D. Pederson, "Simulation program with integrated circuit emphasis," in Midwest Symposium on Circuit Theory, 1973.

[8] K. R. McIntosh. (2018, 02/20/2018). SunSolve. Available: https://www.pvlighthouse.com.au/sunsolve

[9] K. R. McIntosh and S. C. Baker-Finch, "OPAL 2: Rapid optical simulation of silicon solar cells," in Photovoltaic Specialists Conference (PVSC), 2012 38th IEEE, 2012, pp. 000265-000271: IEEE.

[10] F. Dimroth et al., "Wafer bonded four-junction GaInP/GaAs//GaInAsP/GaInAs concentrator solar cells with 44.7\% efficiency," Progress in Photovoltaics: Research and Applications, vol. 22, no. 3, pp. 277-282, 2014. 